\title{
Narrativa Transmedia y Comprensión Lectora: Una experiencia en la Educación Rural Colombiana
}

\author{
Transmedia Narrative and Reading Comprehension: An Experience in Colombian \\ Rural Education
}

Marisol Rodríguez-Silva ${ }^{1}$

\section{ว

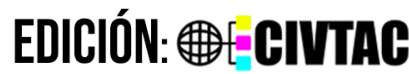

Recibido: 30/diciembre/2020

Aceptado: 8/abril/2021

Publicado: 16/abril/2021

4aís

${ }^{1}$ Colombia

\section{IIIII Institución}

${ }^{1}$ Secretaría de Educación de Cundinamarca

\section{QCorreo Eletrónico \\ 1'marisolrodriguezsilva1987@gmail.com}

\section{iD ORCID}

'https://orcid.org/0000-0001-9568-4449

\section{Citar así: C APA / IEEE}

Rodríguez-Silva, M. (2021). Narrativa Transmedia y Comprensión Lectora: Una experiencia en la Educación Rural Colombiana. Revista TecnológicaEducativa Docentes 2.0, 11(1), 110-119. https://doi.org/10.37843/rted.v11i1.199

\section{Rodríguez-Silva, "Narrativa} Transmedia y Comprensión Lectora: Una experiencia en la Educación Rural Colombiana", RTED, vol. 11, n. ${ }^{\circ} 1$, pp. 110-119, abr. 2021

\section{Resumen}

Hablar de transmedia implica hallarse inmerso en pantallas, libros, música, redes sociales, cine, videojuegos, televisAlfabetización Digital como Puente de Exclusión Social Poscovid-19 panorama lector cada vez más extenso. Esta investigación se desarrolló como un estudio de caso en la IED República de Francia-Escuela San Luis. El objetivo fue evaluar el impacto de la Narrativa Transmedia en la comprensión lectora crítica de estudiantes de grado quinto, quienes se educan en zona rural con el modelo pedagógico Escuela Nueva. Desde un enfoque cualitativo, se usó la etnografía virtual para recolectar datos al aplicar una intervención didáctica diseñada desde las competencias transmedia y cinco dimensiones de comprensión lectora crítica: textual evidente, relacional intertextual, enunciativa, valorativa y sociocultural. Transmediando leyendas colombianas en medios, plataformas, lenguajes y formatos diversos, los estudiantes mostraron como primer resultado el fortalecimiento de las competencias correspondientes a lo narrativo, gestión de contenido, gestión social, uso de medios y tecnología. Adicionalmente, evidenciaron progresos en su comprensión lectora crítica al identificar diferencias entre dos o más textos (dimensión relacional intertextual), comprender intencionalidades subyacentes a las historias (dimensión enunciativa), así como también, reconocer algunos estereotipos o hechos violentos dentro de estas (dimensión sociocultural). Por consiguiente, se concluye que la Narrativa Transmedia impactó positivamente la comprensión lectora crítica de los estudiantes ampliando el panorama lector y generando múltiples oportunidades para expresar los aprendizajes, fortaleciendo así, los principios esenciales del modelo pedagógico Escuela Nueva.

Palabras clave: Narrativa transmedia, Comprensión lectora, Educación rural, Escuela Nueva.

\section{Abstract}

Talking about transmedia implies being immersed in screens, books, music, social networks, cinema, video games, television, and many other possibilities, making it necessary to recognize an increasingly extensive reading panorama. This research was developed as a case study in the IED Republican de Francia-Escuela San Luis. The objective was to evaluate the impact of the Transmedia Narrative on the critical reading comprehension of fifth-grade students who are educated in rural areas with the model New School. From a qualitative approach, virtual ethnography was used to collect data by applying a didactic intervention designed from transmedia skills and five dimensions of critical reading comprehension: evident textual, intertextual relational, enunciative, evaluative, and sociocultural. Transmediating Colombian legends in various media, platforms, languages, and formats, the students showed as a first result the strengthening of the competencies corresponding to the narrative, content management, social management, use of media, and technology. Additionally, they evidenced progress in their critical reading comprehension by identifying differences between two or more texts (intertextual relational dimension), understanding underlying intentions to the stories (enunciative dimension), as well as recognizing some stereotypes or violent events within them (sociocultural dimension). Consequently, it is concluded that the Transmedia Narrative positively impacted the students' critical reading comprehension, expanding the reading panorama and generating multiple opportunities to express learning, thus strengthening the essential principles of the Escuela Nueva pedagogical model. 


\section{Introducción}

Hablar de transmedia implica hallarse inmerso en pantallas, libros, música, redes sociales, cine, videojuegos, televisión, entre muchas otras posibilidades, hace necesario reconocer un panorama lector cada vez más extenso. Lo anterior, porque el texto -durante siglos confinado en páginas de papel-, ha traspasado los límites enriqueciéndose con multiplicidad de interacciones ofrecidas por las pantallas. Ahora bien, como lo explica Albarello (2019), tales interacciones generan prácticas lectoras novedosas: ubicuas, extensivas, relacionales y conectivas. Lo ubicuo se da cuando el lector encuentra las pantallas en cualquier momento o lugar de la vida cotidiana; lo extensivo, se refiere a comprender superficialmente la información saltando velozmente entre textos y dispositivos; lo relacional, pone en juego la capacidad para interconectar los estímulos, los datos; lo conectivo, promueve interacciones con otros lectores.

De igual manera, el texto en su naturaleza tradicional también ha sufrido transformaciones. Ya no conserva un carácter estático conservado en un libro, por el contrario, a través de las pantallas y la variedad mediática permite al lector modificar su contenido constantemente convirtiéndose así en un prosumidor (Albarello, 2019). Según Islas-Carmona (2008), el prosumidor es un actor comunicativo emergente en la sociedad de la ubicuidad y su rol, lejos de lo pasivo, se encamina hacia la autonomía con la cual consume y produce contenidos. Sin embargo, no es contando lo mismo una y otra vez, sino expandiendo, ampliando, reconstruyendo una narrativa con nuevas piezas textuales que un contenido deja de ser sólo un texto a ser todo un universo narrativo, en resumen, una Narrativa Transmedia (Scolari, 2014).

$\mathrm{Al}$

respecto,

Scolari

$(2013 ; 2014 ; 2017 ; 2018)$ define esta narrativa como una historia expandida a través de medios, géneros y plataformas múltiples: los libros, el cine, televisión, las redes sociales, las revistas de comic, los videojuegos, el teatro, los videos, música, entre otros. Ahora bien, en décadas anteriores se han presentado estudios en torno a la interacción de los jóvenes con medios de comunicación y tecnología, poniendo especial acento en el ejercicio crítico del lector, o como lo explica López (2003), el propósito de formar "una audiencia inteligente con criterio." (p.9). De nuevos modos, esta preocupación sigue latente en estudios sobre Narrativa Transmedia; en el contexto colombiano, por ejemplo, encontramos investigaciones de Polo (2018), Barreneche et al. (2018), Amador-Baquiro (2018), Rodríguez et al. (2015), el proyecto Profes Transmedia (IDEP, 2018), entre otras. A nivel internacional, es loable la apuesta del proyecto Transmedia Literacy (Scolari, 2018), el cual reunió investigadores de ocho países logrando definir, clasificar y caracterizar competencias transmedia.

En resumen, estas investigaciones ponen en evidencia cómo los jóvenes desarrollan prácticas lectoras y competencias estrechamente ligadas a la Narrativa Transmedia de manera informal, fuera del entorno escolar. Por lo tanto, plantean como necesidad alfabetizar transmedialmente a los jóvenes, haciendo de ellos prosumidores críticos con capacidad para comprender, comparar, cuestionar, analizar, evaluar y comunicar responsablemente.

Esta investigación decide participar en la discusión con un estudio realizado en la IED República de Francia-Escuela Rural San Luis, ubicada en zona rural de Cundinamarca (Colombia). Específicamente, la población estudiada estuvo conformada por estudiantes de grado quinto quienes se educan con el modelo pedagógico Escuela Nueva. Según Colbert (2006), este modelo se fundamenta en los principios de la pedagogía activa y promueve:

un proceso de aprendizaje cooperativo y
personalizado centrado en el alumno, la
formación de valores y comportamientos
democráticos, un nuevo rol del docente como
orientador y facilitador y un nuevo concepto de
textos interactivos o guías de aprendizaje para
lograr así el mejoramiento de la calidad y
efectividad del aprendizaje (p.196).

En cuanto a la situación problema que dio origen a esta investigación, se halló en los estudiantes un bajo desempeño en las evaluaciones de comprensión lectora, con mayor énfasis en el nivel crítico (MEN, 2018). No obstante, también se encontró que el panorama 
lector de los estudiantes era bastante restringido, pues las cartillas, guías y libros de texto se usaban con predominancia, dejando a un lado sus intereses; en contraste, los medios tecnológicos asistían las clases con un carácter más instrumental o auxiliar, desaprovechando su potencial para explorar otras posibilidades lectoras. Para resumir, en palabras de Mujica (2019), el problema evidenciado consiste en un desfase con la realidad de los estudiantes, cuyas formas de comunicación distan bastante de lo presentado en la escuela.

A raíz de lo anterior, esta investigación trazo como objetivo principal, implementar una intervención didáctica transmedia para evaluar su impacto en el fortalecimiento de la comprensión lectora crítica en estudiantes de grado quinto, quienes se educan con el modelo pedagógico Escuela Nueva. Lo anterior, en el contexto específico de la IED República de FranciaEscuela San Luis, una institución de tipo rural en Colombia.

\section{Metodología}

Desde un enfoque predominantemente cualitativo, con apoyo de algunos datos cuantitativos, la investigación se perfila como un estudio de caso al examinar en profundidad una unidad de análisis específica, haciendo parte de un universo poblacional e interactuando en un contexto con unas características propias (Bernal, 2010). En el caso presente, esta unidad es constituida por los estudiantes de grado quinto de la IED República de Francia-Escuela Rural San Luis; sin embargo, al ser un estudio de caso, es pertinente incluir también otras perspectivas: docentes y padres de familia.

Ahora bien, esta investigación debió implementarse -en buena parte- durante el aislamiento obligatorio generado por el COVID 19 en Colombia, por lo que se realizó una adaptación metodológica con el fin de recolectar los datos de manera no presencial, usando los dispositivos tecnológicos disponibles. La etnografía virtual, entonces, se adoptó como técnica facilitadora para actuar en el campo investigativo a través de dispositivos tecnológicos, interactuando con los participantes, observando sus acciones, flujos y experiencias (Hine, 2000).
Teniendo como referente el estudio acerca de etnografía virtual y Narrativa Transmedia realizado por Pink \& Ardévol (2018), se opta por usar una bitácora transmedia con el fin de registrar las acciones realizadas por los estudiantes en cada sesión respecto a los medios o plataformas usados, así como sus aprendizajes. Asimismo, las observaciones, grupos focales, entrevistas, entre otras interacciones, se registraron en el diario de campo para su posterior análisis. Finalmente, se aplicaron dos pruebas de comprensión lectora crítica al iniciar y finalizar la intervención didáctica transmedia, con el fin de evaluar formativamente el proceso de los estudiantes.

La comprensión lectora puede definirse como un "proceso de búsqueda y reconstrucción del significado y sentido que implica cualquier manifestación lingüística." (MEN, 2006, p.21). Sin embargo, según el ICFES (2018), este proceso se manifiesta de manera específica en tres nivel de lectura: el nivel literal, cuando se comprende contenidos específicos dentro del texto (microestructura); lo inferencial, se desarrolla con la construcción del sentido global sobre el texto (macroestructura); el nivel crítico, valoraciones $\mathrm{o}$ evaluaciones del lector relacionando el texto con el contexto, los puntos de vista, así como la carga persuasiva (superestructura).

Al respecto, Cassany (2003) señala este nivel de comprensión como el más alto, exigiendo un desarrollo previo de lo literal e inferencial para llevar al lector a tomar una postura personal sobre el texto, su contenido, las intenciones expresadas de manera explícita e implícita, entre otros. Implica además, como lo explica Ricoeur (1995), no sólo la interacción entre el texto y el lector, sino una "intersección del mundo del texto con el del lector" (Citado por Jurado, 2008, p.151).

En relación a lo anterior, el ICFES (2013) caracteriza cinco dimensiones que configuran la comprensión lectora crítica: textual evidente, relacional intertextual, enunciativa, valorativa y sociocultural. Por lo cual, esta investigación plantea cinco categorías de análisis, cuya definición se corresponde con dichas dimensiones, estableciendo a su vez un diálogo con las competencias transmedia definidas en el proyecto Transmedia Literacy (Scolari, 2018) 
1. Dimensión textual evidente: se refiere a la comprensión básica del texto a partir de los niveles literal e inferencial, pues el lector logra entender los tópicos fundamentales. Respecto a los textos continuos, esta dimensión también implica manejar los recursos anexos al texto: índices, notas al pie, imágenes, símbolos, entre otros; respecto a los textos discontinuos esta dimensión involucra el manejo de los dispositivos tecnológicos que alojan un texto (competencias sobre uso de medios y tecnologías), la gestión de su búsqueda, rastreo, ubicación o manejo (competencias sobre gestión de contenido), así como el conocimiento para usar imagen, audio y video al acceder al texto (competencias de producción).

2. Dimensión relacional intertextual: En referencia a los textos continuos, se refiere a cómo se relacionan las partes que componen un texto, las ideas allí expuestas, los puntos de encuentro con otros textos; en referencia a los textos discontinuos, esta dimensión ejercita la forma de comprender cómo se relacionan diversas piezas textuales en un universo narrativo mientras se navega a través de diversos dispositivos, medios, plataformas y géneros (competencias narrativas), permite inspirarse en las producciones de otros para crear nuevas (competencias de producción), así como también lleva a contrastar fuentes de información (competencias sobre prevención de riesgos, ética e ideología).

3. Dimensión enunciativa: comprende intenciones o propósitos tras el texto y los participantes de la situación comunicativa. Tanto en textos continuos como discontinuos, exige reconocer tipología textual, géneros narrativos, así como variedad de formatos (competencias narrativas); también implica la forma de comprender $\mathrm{o}$ producir un texto en compañía de otros, para lo cual se comparten los conocimientos a modo de enseñanza aprendizaje y se distribuyen funciones de producción -cámaras, guion, etcétera(competencias de gestión social).

4. Dimensión valorativa: Tanto en textos continuos como discontinuos, se hacen apreciaciones estéticas sobre los textos tomando decisiones de consumo a partir de ellas (competencias narrativas); se expresan los puntos de vista sobre el texto haciendo recomendaciones a otros (competencias de gestión social); se valora también el criterio para producir textos definiendo cuáles son los formatos $o$ géneros con los cuales se obtienen mejores contenidos (competencias sobre uso de medios y tecnología); se lidia con la frustración reconociendo la necesidad de pedir ayuda a otros (competencias de gestión individual); se revisan las producciones de otros para apropiarlas haciéndoles modificaciones o mejoras (competencias de producción); se toma una postura crítica sobre el por qué se lee determinado texto o contenido y se reflexiona sobre sus riesgos (competencias sobre prevención de riesgos, ética e ideología).

5. Dimensión sociocultural: cualquier texto, sea continuo o discontinuo, se da en un contexto determinado por condiciones históricas, sociales y culturales. Por lo tanto, el propósito del lector debe encaminarse hacia el fortalecimiento de una postura crítica que le permita evaluar el discurso, la carga ideológica, violencia, estereotipos, estrategias publicitarias, intenciones de los medios de comunicación, entre otros (competencias sobre prevención de riesgos, ética e ideología). Esta dimensión también comprende el código de comportamiento según el entorno digital explorado por el lector, así como su capacidad de crear colaborativamente (competencias de gestión social) produciendo textos de acuerdo con una audiencia específica (competencias de producción).

Como muestra la Tabla 1, esta investigación se desarrolló en tres fases. El momento o fase inicial, tuvo como objetivo reconocer las concepciones de padres y 
estudiantes en torno a lo que significa leer en la Escuela Nueva, así como evaluar a modo diagnóstico la comprensión lectora crítica de los estudiantes. Por lo anterior, el primer paso consistió en desarrollar un grupo focal en el que los participantes socializaron sus experiencias, prácticas e ideas.

\section{Tabla 1}

Fases de investigación "Narrativa Transmedia para el fortalecimiento de la comprensión lectora en la Escuela Nueva. Una experiencia de aula en la IED República de Francia"

\begin{tabular}{|c|c|}
\hline \multicolumn{2}{|c|}{ Momento } \\
\hline \multirow[b]{2}{*}{ 承 } & Grupo focal 1. \\
\hline & $\begin{array}{l}\text { Evaluación de comprensión lectora crítica } \\
\text { (inicial) }\end{array}$ \\
\hline \multirow{5}{*}{ 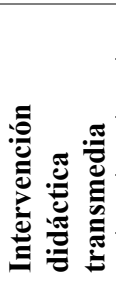 } & Unidad 1. Dimensión textual evidente. \\
\hline & Unidad 2. Dimensión relacional intertextual. \\
\hline & Unidad 3. Dimensión enunciativa. \\
\hline & Unidad 4. Dimensión valorativa. \\
\hline & Unidad 5. Dimensión sociocultural. \\
\hline \multirow{2}{*}{ 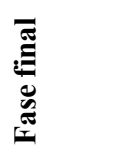 } & $\begin{array}{l}\text { Evaluación de comprensión lectora crítica } \\
\text { (final). }\end{array}$ \\
\hline & Grupo focal 2. \\
\hline
\end{tabular}

Nota. Fases desarrolladas en el estudio que dio origen a este artículo, elaboración propia (2020).

Posteriormente, se aplicó una evaluación orientada a observar el desempeño de los estudiantes en cada una de las categorías de análisis planteadas en el trabajo de investigación: textual evidente, relacional intertextual, enunciativa, valorativa y sociocultural. Se diseñó con un total de 23 preguntas divididas en cinco secciones, usando textos de tipo continuo y discontinuo, acudiendo a formatos, géneros, plataformas variadas, así como a preguntas cerradas y abiertas. Para acotar, previamente los estudiantes eligieron las leyendas como temática a transmediar, por lo cual la evaluación inicial se diseñó en torno a "La Patasola", la final tuvo como temática "El Mohan".

En una segunda etapa, se planteó una intervención didáctica transmedia estructurada, nuevamente, en cinco unidades que se corresponden con las categorías de análisis mencionadas anteriormente. De manera concreta, las actividades que realizaron los estudiantes se describen a continuación:

1. Unidad didáctica 1: los estudiantes rastrearon en la web una leyenda de su preferencia mientras navegaron por distintos medios, plataformas, formatos y géneros. Posteriormente socializaron la leyenda elegida mediante un formato de libre elección: comic, presentación animada, video, historia escrita.

2. Unidad didáctica 2: intercambiaron con sus compañeros links y contenidos variados acerca de las leyendas, luego contrastaron diferentes versiones escritas para construir un cuadro comparativo determinando semejanzas y diferencias.

3. Unidad didáctica 3: elaboraron un podcast sobre las intenciones de las leyendas, así como los personajes que intervienen en ellas. Para lograrlo entrevistaron a un familiar a partir de algunas preguntas orientadoras y usaron recursos auditivos adicionales para ambientarla.

4. Unidad didáctica 4: crearon una expansión narrativa a partir de una leyenda colombiana a modo de precuela, secuela o historia paralela. Además, la socializaron en formato de libre elección: presentación animada, podcast, comic, video, texto escrito.

5. Unidad didáctica 5: participaron en un conversatorio con el cual reflexionaron sobre la influencia de las leyendas en su contexto cercano en cuanto a la carga de violencia, estereotipos, enseñanzas, función y propósito. De igual manera, expresaron los aprendizajes logrados durante el proceso identificando sus puntos fuertes y aspectos a mejorar.

Finalmente, se aplicó una segunda evaluación de comprensión lectora crítica cuya estructura es igual a la primera, asimismo, permitió evidenciar en cuáles dimensiones de este nivel lector, así como las competencias transmedia, habían progresado los estudiantes. También, se desarrolló el grupo focal final con el cual los participantes conversaron nuevamente sobre sus prácticas lectoras, el aprendizaje 
logrado y los cambios observados durante el proceso.

\section{Resultados}

Respecto a las competencias narrativas, se evidenció cómo los estudiantes se acercaron espontáneamente a las leyendas desde una lectura intertextual, buscaron diferentes versiones navegando a través de medios, formatos, géneros variados; además, en lugar de profundizar o analizar una leyenda, prefirieron migrar entre ellas realizando contenidos variados, lo que denota buena práctica de la lectura extensiva.

Además, al abordar una historia buscaban hallar relaciones o conexiones con otros textos de tipo audiovisual para comprenderla, lo cual pone de manifiesto una lectura de tipo relacional. Esto último también se refleja en las producciones transmedia elaboradas por los estudiantes porque consiguieron hallar puntos de encuentro y desencuentro entre las leyendas, así como también las expandieron creando secuelas, precuelas o historias paralelas aportando hechos o datos novedosos al universo narrativo.

Respecto a las competencias de gestión de contenido, los estudiantes ejercitaron ampliamente el rastreo de contenido en la web para hallar piezas textuales acerca de las leyendas. Además, mejoraron la forma de compartir sus hallazgos y socializarlos -tanto con sus compañeros como docente- a través de YouTube, Facebook, Whatsapp, Google Forms, Google Meet, entre otros. En cuanto a las competencias de producción, los estudiantes exploraron herramientas digitales desconocidas o mejoraron el manejo de aquellas habitualmente usadas. Por ejemplo, fortalecieron el uso de Power Point, se adentraron en Genially (https://www.genial.ly/es), aprendieron a usar Comica (Game Brain, 2020), así como también fortalecieron el uso del grabador de voz, cámara para elaborar podcast, entre otros.

Para resaltar, los estudiantes mostraron prácticas lectoras asociadas a lo conectivo a partir de la socialización de textos porque, gracias al intercambio de valoraciones entre pares, reconocieron aquellos medios y formatos con los cuales se les facilitaba crear contenidos, pero también aquellos que no eran su fuerte. A su vez, lo anterior les permitió adquirir mayor criterio para proponer actividades o textos relacionados con lo transmedial en clases distintas a lenguaje. En referencia a las competencias sobre uso de medios y tecnologías, es destacable la recursividad de los participantes en el desarrollo de la intervención didáctica transmedia, usando los recursos disponibles en su hogar para investigar, explorar, aprender y crear pese a las limitaciones de conectividad que se acentuaron durante el aislamiento obligatorio del año 2020 en Colombia.

En relación con las competencias de gestión social, es sobresaliente la integración de las familias en las producciones transmedia de los estudiantes. Los tíos, hermanos o primos mayores fueron relevantes en el desarrollo de esta competencia porque en medio del aislamiento compartieron sus conocimientos sobre formatos, aplicaciones y medios con los estudiantes, además de participar en entrevistas, videos, entre otras producciones. En cuanto a las competencias sobre prevención de riesgos, ética e ideología se evidenció un desarrollo leve; aunque los estudiantes -después de conversaciones reflexivas- exponían sus opiniones sobre las leyendas reconociendo algunos hechos violentos y mencionando algunos estereotipos, les costaba identificar cómo esto puede afectar al lector o su contexto cercano.

Finalmente, en el ámbito pedagógico, es importante resaltar que se fortalecieron los principios esenciales de la Escuela Nueva. La experiencia natural, por ejemplo, se promovió en el ejercicio espontáneo de las prácticas lectoras extensivas, relacionales y conectivas; el principio de actividad se dinamizó cuando los estudiantes -en tanto prosumidores- decidieron cuál leyenda abordar, su formato de presentación, plan de expansión; asimismo, esta capacidad para decidir dentro de su proceso de aprendizaje fortaleció también los principios de motivación $\mathrm{y}$ autonomía.

\section{De las evaluaciones de comprensión lectora crítica en relación con las competencias transmedia}

Como se observa en la Tabla 2, en las evaluaciones inicial y final, los estudiantes obtuvieron un promedio total de 17 y 21,86 
respectivamente. Lo anterior, muestra un progreso del $28,57 \%$ impactando positivamente el aspecto comprensión lectora crítica en los estudiantes. En cuanto a lo sucedido con cada una de las dimensiones, así como los aspectos específicos en los cuales mejoró el grupo, se expondrá a continuación.

\section{Tabla 2}

Resultados evaluaciones de comprensión lectora crítica en relación con competencias transmedia, aplicada a estudiantes de grado $5^{\circ}$ de la IED República de Francia-Escuela Rural San Luis

Resultados Evaluaciones de

Comprensión Lectora Crítica

\begin{tabular}{|c|c|c|c|}
\hline Dimensión & $\begin{array}{l}\text { Ev. } \\
\text { Inicial }\end{array}$ & $\begin{array}{l}\text { Ev. } \\
\text { Final }\end{array}$ & $\begin{array}{l}\text { \%Progre } \\
\text { so }\end{array}$ \\
\hline $\begin{array}{l}\text { Textual } \\
\text { Evidente }\end{array}$ & 5,00 & 6,00 & $20 \%$ \\
\hline Relacional Int. & 3,14 & 4,43 & $41 \%$ \\
\hline Enunciativa & 3,86 & 5,14 & $33 \%$ \\
\hline Valorativa & 3,57 & 4,00 & $12 \%$ \\
\hline Sociocultural & 1,30 & 2,30 & $76,9 \%$ \\
\hline $\begin{array}{l}\text { Promedio } \\
\text { Total } \\
\text { Evaluación }\end{array}$ & 17,00 & 21,86 & $28,57 \%$ \\
\hline
\end{tabular}

Nota. Se muestra el promedio obtenido por el grupo de estudiantes en cada dimensión, tanto en la evaluación inicial como final. El promedio máximo en cada dimensión es de 8 , por lo tanto, el puntaje máximo para cada evaluación es de 40 puntos; en el \% de progreso se muestra cuánto avanzó el grupo en la evaluación final, respecto de la inicial, elaboración propia (2020).

En cuanto a lo textual evidente, el grupo progresó un $20 \%$ logrando identificar con mayor facilidad el contenido literal en textos continuos, como por ejemplo las leyendas escritas, y lo inferencial en textos discontinuos como los videos, el comic, el podcast, entre otros. El proceso relacional intertextual alcanzó un progreso del $41 \%$, mostrando en los estudiantes mayor habilidad para hallar diferencias, mas no semejanzas, entre textos de tipo continuo y discontinuo. En contraste, persisten dificultades al comprender relaciones internas del texto, así como también la función de sus diferentes partes.

Respecto lo enunciativo, los estudiantes alcanzaron un progreso del $33 \%$. Es destacable el avance del grupo en cuanto al reconocimiento de la intención de las leyendas y sus personajes en textos discontinuos como el podcast, videos, entre otros textos audiovisuales.
Llamativamente, por el contrario, esto mismo se manifiesta como dificultad cuando el texto es netamente continuo o escrito.

En el aspecto sociocultural se presentó el mayor progreso: 76,09\%. Aunque fue la dimensión con mayor avance, los resultados finales fueron los más bajos con un promedio de 2.30 , pues pese a reconocer algunos estereotipos $\mathrm{y}$ hacer algunas afirmaciones sobre cómo las leyendas influencian su contexto cercano, los argumentos o justificaciones que exponen son aún simples. Algo similar sucedió con la dimensión valorativa; se dio un progreso del $12 \%$ pero al expresar valoraciones sobre las leyendas, las justificaciones son también simples.

De los grupos focales

Los padres de familia ampliaron sus concepciones en torno a la lectura en su experiencia con el modelo pedagógico Escuela Nueva. El primer grupo focal, permitió descubrir una noción de lectura desde los libros de textos escolares, la literatura infantil y los textos escritos o continuos; el segundo grupo focal, mostró algunos cambios enriquecedores en los padres, quienes señalaban en la lectura posibilidades tan variadas como los videos, las imágenes y la la web. De igual manera, reconocieron el internet y otras herramientas tecnológicas como algo indispensable en la actualidad, por consiguiente, manifestaron también la imperante necesidad de incluirlos en la educación de sus hijos con el fin de facilitarles explorar otros tipos de lectura.

Respecto a los cambios observados en los estudiantes durante el proceso de intervención didáctica transmedia, los padres manifestaron que los estudiantes habían conocido diferentes versiones de las leyendas, por tal razón, ahora podían expresar fácilmente semejanzas o diferencias entre las historias. Adicionalmente, como aspectos más resaltantes se encuentran los procesos de expresión corporal, oral, comunicación audiovisual.

Por otra parte, destacaron cómo tras la intervención didáctica transmedia los estudiantes lograron demostrar sus aprendizajes de maneras variadas superando las barreras de los textos tradicionales. De hecho, los padres de algunos estudiantes cuya historia escolar había sido marcada por las dificultades académicas, señalaban como una ventaja expresar lo 
aprendido a través de videos, podcast, presentaciones, maquetas, escritos y más. Como se observa, esta narrativa favorece el respeto por el desarrollo progresivo e individual de los estudiantes, permitiéndoles aprender a su propio ritmo mediante una actividad enriquecida por las múltiples opciones transmediales. Lo anterior, cabe señalar, coincide con los principios fundamentales de la Escuela Nueva (Flórez, 1995).

Finalmente, como dificultades sobresalientes durante la intervención didáctica transmedia, los participantes manifestaron su inconformidad en cuanto a la recepción inestable de la señal que se da en algunos sectores rurales, el acceso limitado a paquetes de datos por dificultades económicas y bastantes restricciones de movilidad vivenciadas en el aislamiento obligatorio.

\section{Conclusiones}

De manera espontánea, los estudiantes mostraron prácticas lectoras asociadas a lo extensivo, relacional y conectivo. Como se evidenció, migraron entre textos, formatos, géneros $\mathrm{y}$ plataformas buscando encontrar conexiones o relaciones que les permitieran comprender con mayor facilidad una historia. No obstante, evitaron profundizar en un único relato explorando diferentes versiones; además, el intercambio social entre pares les permitió establecer criterios respecto a la creación de contenidos.

La Narrativa Transmedia favoreció el proceso de comprensión literal a partir de textos continuos y lo inferencial a partir de textos discontinuos (dimensión textual evidente). Además, potenció significativamente el reconocimiento de diferencias entre dos o más textos provenientes de géneros $\mathrm{o}$ formatos variados (dimensión relacional intertextual); contribuyó también al reconocimiento de intencionalidades, tanto de los personajes como de las historias, con mayor énfasis en textos discontinuos como el podcast, comics, entre otros (dimensión enunciativa). Finalmente, aunque generó oportunidades de reflexión llevando a los estudiantes a identificar algunos aspectos sobre violencia y estereotipos en los relatos, es necesario ejercitar aún más la capacidad argumentativa.

Por otra parte, las competencias transmedia cuyo desarrollo fue sobresaliente corresponden a lo narrativo, gestión de contenido, gestión social, uso de medios y tecnología. Esto se evidenció, en gran parte, en la creación de expansiones narrativas que los estudiantes elaboraron en torno a las leyendas, además de los aprendizajes logrados al rastrear, ubicar, compartir y socializar contenidos en formatos diversos, usando medios tecnológicos disponibles, aprendiendo de los pares, así como también de los familiares mayores.

En definitiva, la investigación concluye finalmente que la Narrativa Transmedia impactó positivamente la comprensión lectora crítica de los estudiantes, a partir de una intervención didáctica estructurada desde cinco dimensiones que configuran este nivel lector (ICFES, 2013) y el ejercicio de competencias transmedia definidas en el proyecto Transmedia Literacy (Scolari, 2018). De lo anterior, sobresalen las dimensiones intertextual y enunciativa, con los mayores progresos, así como lo textual evidente por su consolidación y óptimos resultados. Asimismo, se destacan las competencias referentes a lo narrativo, gestión de contenido, gestión social, uso de medios y tecnología.

Por último, desde un punto de vista pedagógico, es imprescindible señalar que esta narrativa enriquece la Escuela Nueva ampliando el panorama lector a partir de interacciones con textos en distintos formatos, medios, lenguajes, géneros y plataformas. A su vez, genera múltiples posibilidades, tanto para desarrollar como para expresar aprendizajes, fortaleciendo así la experiencia natural, actividad, motivación, autonomía, respeto por el desarrollo progresivo e individual, principios fundamentales de este modelo educativo.

\section{Reconocimiento}

Agradecimientos al Doctor Jaime Alejandro Rodríguez y la Maestría en Educación en Tecnología de la Universidad Distrital Francisco José de Caldas. 


\section{Referencias}

Albarello, F. (2019). Lectura Transmedia: Leer, escribir, conversar en el ecosistema de pantallas. Editorial Ampersand.

Amador-Baquiro, J. (2018). Educación interactiva a través de narrativas transmedia: posibilidades en la escuela. Revista Magis,10 (21), 77-94. https://doi.org/10.11144/Javeriana.m10-21.eint

Barreneche, C., Polo, N., \& Menéndez, A. (2018). Alfabetismos transmedia en Colombia: estrategias de aprendizaje informal en jóvenes gamers en contextos de precariedad. Revista Chasqui, (137), 169-187. https://doi.org/10.16921/chasqui.v0i137.3510

Bernal, C. A. (2010). Metodología de la Investigación. Pearson Education.

https://www.academia.edu/25497606/Metodología_de_1 a_Investigación?auto=download

Cassany, D. (2003). Aproximaciones a la lectura crítica: teoría, ejemplos y reflexiones. Tarbiya, (32), 113-132. https://revistas.uam.es/tarbiya/article/viewFile/7275/762 3

Colbert, V. (2006). Mejorar la calidad de la educación en escuelas de escasos recursos. El caso de la Escuela Nueva en Colombia. Revista Colombiana de Educación, (51), 186212.

http://www.redalyc.org:9081/articulo.oa?id=413635245 008

Ferrés, J. (2000). Educar en una cultura del espectáculo. Paidós, Editorial. https://marciate18.files.wordpress.com/2013/09/metafor a_del_navegante_2_.pdf

Flórez, R. (1995). La dimensión pedagógica-Formación y Escuela Nueva en Colombia. Revista Educación y $\left.\begin{array}{lllll}\text { Pedagogía, } & 7 & (14 & \text { y } & 15\end{array}\right), \quad 197-219$. http://aprendeenlinea.udea.edu.co/revistas/index.php/rev istaeyp/article/view/5588

Game Brain, (2020). Comica [Aplicación móvil]. https://play.google.com/store/apps/details?id=gr.gamebr ain.comica\&hl=es_CO

Hine, C. (2000). Etnografia virtual. Editorial UOC. https://seminariosocioantropologia.files.wordpress.com/ 2014/03/hine-christine-etnografia-virtual-uoc.pdf

Instituto Colombiano para la Evaluación de la Educación (2013). Guías-Módulo Lectura Crítica. https://www.unitecnar.edu.co/sites/default/files/pdfs/Mó dulo-LECTURA

CRITICA.pdf?fbclid=IwAR3EprMv0HQHCPkZc8CzN adCWjOCqxeBuKpRbltCgRLvjTBA-9gFFVwCeJQ

Instituto Colombiano para la Evaluación de la Educación (2018). Marco de referencia para la evaluación, ICFES. Módulo Lectura Critica. https://www.icfes.gov.co/documents/20143/1479027/Ma rco de referencia - lectura critica.pdf

Instituto para la Investigación Educativa y el Desarrollo Pedagógico (2018). Profes transmedia. Docentes en búsqueda de narrativas para la apropiación social del conocimiento. Taller de Edición Rocca S.A.S. http://www.idep.edu.co/sites/default/files/libros/LIBRO PROFES_TRANSMEDIA.pdf

Islas-Carmona, J. (2008). El prosumidor. El actor comunicativo de la sociedad de la ubicuidad. Revista PalabraClave,11(1),29-39.

https://palabraclave.unisabana.edu.co/index.php/palabra clave/article/view/1413

Jurado, F. (2008). La formación de lectores críticos desde el aula (46). Revista Iberoamericana de Educación. https://rieoei.org/historico/documentos/rie46a05.htm

López, D. (2003). El consumo crítico de los medios de la juventud y el lenguaje de la discreción como propuesta pedagógica. Revista Palabra-Clave, 9, 2003, https://palabraclave.unisabana.edu.co/index.php/palabracl ave/article/view/424

Ministerio de Educación Nacional de Colombia (2006). Estándares Básicos de Competencias en Lenguaje, Matemáticas, Ciencias y Ciudadanas. Editorial Escribe y Edita. https://www.mineducacion.gov.co/1621/articles340021_recurso_1.pdf

Ministerio de Educación Nacional de Colombia (2018). Reporte de la Excelencia 2018. Colombia. https://diae.mineducacion.gov.co/dia e/siempre diae/do cumentos/2018/125658000231.pdf

Moreno, J., Ayala, R., Díaz, J. y Vásquez, C. (2010). Prácticas lectoras: comprensión y evaluación. Tendencias, estado y proyecciones. Forma y Función, 23(1). http://www.scielo.org.co/scielo.php?script=sci_arttext\& pid $=$ S0120-338X2010000100007\&lang=es

Mujica, R. (2019). El cambio del estudiante ante la tecnología Revista Tecnológica-Educativa Docentes 2.0, 4(4), 8-9. https://ojs.docentes20.com/index.php/revistadocentes20/article/view/58

Pink, S., \& Ardévol, E. (2018). Estrategias etnográficas para revelar prácticas transmedia de los adolescentes. En Scolari, C. (Ed.), Adolescentes, medios de comunicación $y$ culturas colaborativas. Aprovechando las competencias transmedia de los jóvenes en el aula (pp.114-124).

http://transmedialiteracy.upf.edu/sites/default/files/files/ TL Teens es.pdf

Polo, N. (2018). Lectoescritura juvenil en tiempos de narraciones transmedia. Comunicación y Sociedad, 33, 41-64. http://www.scielo.org.mx/pdf/comso/n33/0188-252Xcomso-33-41.pdf

Rodríguez, J., López, L., \& González, L. (2015). La narrativa transmedia como experiencia de simulación de inteligencia colectiva. El caso de Atrapados. Signo y Pensamiento, 34(67), 60-74. https://doi.org/10.11144/Javeriana.syp34-67.ntes

Scolari, C. (2013). Narrativas transmedia: cuando todos los medios cuentan. https://www.fadu.edu.uy/esteticadiseno-ii/files/2013/05/119756745-1r-CapituloNarrativas-Transmedia.pdf

Scolari, C. (2014). Narrativas transmedia: nuevas formas de 
comunicar en la era digital. Anuario AC/E de cultura digital, $\quad$ Tema $\quad 6 . \quad 71-81$ https://www.accioncultural.es/media/Default $\% 20$ Files/a ctiv/2014/Adj/Anuario_ACE_2014/6Transmedia_CScol ari.pdf

Scolari, C. (2017). El translector. Lectura y narrativas transmedia en la nueva ecología de la comunicación. En Millán, J. (Ed.) La lectura en España. Informe 2017, 175 186.

http://www.fge.es/lalectura/docs/La_Lectura_en_Espana .pdf

Scolari, C. (Ed.) (2018). Adolescentes, medios de comunicación $y$ culturas colaborativas. Aprovechando las competencias transmedia de los jóvenes en el aula. http://transmedialiteracy.upf.edu/sites/default/files/files/ TL_Teens_es.pdf 